\title{
Aggregation Processes Modeling in Physical-Chemical Systems
}

\author{
Naukenova Aigul Sagindykovna, Bekaulova Aliya Amankulovna, Mamitova Aigul Dzhanabaevna, Sadykov \\ Zhenis Abzhanovich, Kerimbekova Zaurekul Maidanbekovna, Tolegen Marzhankul Erhozhakyzy, Ramatullaeva \\ Lazzat Immamadinovna and Tursynbekova Elmira Nurlankyzy \\ M.Auezov South Kazakhstan State University, 160012, Shymkent, Kazakhstan
}

Received: October 09, 2013 / Accepted: October 30, 2013 / Published: December 25, 2013.

\begin{abstract}
In article, the methodology of mathematical modeling of periodic reactors with agitating in the conditions of formation and sedimentation of insoluble solid phase in a working zone on the basis of diffusive model is suggested. Also equations for calculation of the general concentration and average order of clusters of insoluble phase in the periodic reactor are offered. The methodology of calculation of processes of insoluble solid phase aggregation in systems with chemical reactions of the first and pseudo-first orders is developed, namely: equation for calculation of evolution of total clusters' number and average order of globules in system are developed, by taking into account kinetic constants of chemical reaction and elements of aggregation matrix.
\end{abstract}

Key words: Monomer, cluster, chemical source, model time, nucleation.

\section{Introduction}

The article considers with the problems of modeling the aggregation of disperse phase in the reacting suspensions. Many processes of chemical technologies proceed in difficult disperse systems, or are accompanied by formation of disperse firm phase during the process. These are processes proceeding in chemical reactors with formation of insoluble phase, processes accompanied by condensation in dust-gas systems and formation of slimes etc. Existing now methods of calculation and designing of chemical-technological processes and devices with formed or transformed disperse systems (suspensions, emulsions, gels etc.) are poorly developed. All known theoretical and experimental researches consider disperse systems in which the fast chemical transformations leading to formation of new disperse phases do not proceed. Calculation and designing of

Corresponding author: Mamitova Aigul Dzhanabaevna, candidate of technical science, research field: environmental protection. E-mail: a-mamitova@mail.ru. reactors with formation of insoluble disperse phase in volume of device was spent by the same methods, as calculation and designing of reactors with homogeneous or pseudo-homogeneous mediums, and account of influence of insoluble disperse phase was come down to corrections that lead to essential errors at calculation and designing of devices, or demanded carrying out of special experimental researches with taking into account the scales of real industrial production. Below some processes in which such situation can take place is shown: first of all, it is large-capacity manufactures of dicalciumphosphates in the phosphoric industry and manufacture of polystimulin A- 6 by copolymerisation of acrylic acid and allylic ether of 2.4 dichlorophenoxyacetic acids. Questions of modelling of reactors for these processes have been deeply studied by Yeskendirov [1] who in his researches has clearly shown essential influence of processes of nucleation rate and sedimentation on structure of streams in the device. Thus the concept of calculation which takes into account the noted 
phenomena is not finished. Further, it is necessary to note processes of creation of composite materials by mixing of various components with structural and phase transformations in system, processes of coagulation in systems of water preparation and many other processes.

For describing the evolution of the $i$-mers concentration in the apparatus we can use various modifications of Smoluchowski or Becker-Döring equations expanded by the term which corresponds to the source of mass. This source occurs as a result of insoluble products of chemical reactions.

\section{Theoretical Details and Discussion}

It was assumed that main contribution to the aggregation process is conditioned by diffusion limited aggregation (DLA) [2, 3]. Thus taking into account the aggregation and fragmentation phenomena we can use the Becker-Döring equations describing the dynamics of $i$-mers concentration in the form:

$$
\begin{gathered}
S\left(C_{i}\right)=\frac{\partial C_{i}}{\partial t}=J_{i-1}(C)-J_{i}(C)+\Gamma_{i}, \\
\frac{d C}{d t}=-J_{1}(C)-\sum_{i=1}^{\infty} J_{i}(C)+\Gamma_{1},
\end{gathered}
$$

where vector $C=\left(C_{i}\right)$, and $\Gamma_{i}$ is the chemical source of $i$-mers.

All components of fluxes in the Becker-Döring equations for $i \geq 2$ can be written in the following form $[3,4]$ :

$$
\begin{gathered}
J_{1}(C)=a_{i} C_{1} C_{i}-b_{i+1} C_{i+1}, \\
\frac{d C_{i}}{d t}=\frac{1}{2} \sum_{s=1}^{i-1} a_{s, i-s} C_{S} C_{i-s}-C_{i} \sum_{i s=1}^{\infty} a_{i, s} C_{S} \\
+\sum_{s=i+1}^{\infty} b_{s},{ }_{i} C_{S}-\frac{C_{i}}{i} \sum_{s=1}^{i-1} s b_{i, s}
\end{gathered}
$$

Aggregation without chemical reactions is determined the local mass conservation law for particles consisting from finite number of origin particles- embryos.

In this case, the following balance equation is correct

$$
\sum_{i} i S\left(C_{i}\right)=0
$$

In the presence of chemical reactions in the system the balance equation reads

$$
\sum_{i} i S\left(C_{i}\right)=-\sum_{i} i \Gamma_{i}
$$

If there are not the fragmentation phenomena in the system then the Becker-Döring equation transforms to the Smoluchowski equation adapted for binary coagulation.

$$
\frac{d C_{i}}{d t}=\frac{1}{2} \sum_{j=1}^{i-1} \Phi_{i-j, j} C_{i-j} C_{j}-C_{i} \sum_{j=1}^{\infty} \Phi_{i, j} C_{j}
$$

As it is shown in Ref. [2] the most likely mechanism of aggregation-fragmentation is the binary interaction. Thus the function of $i$ - and $j$-mers coagulation reads:

$$
a_{i, j}=\sigma_{i, j}\left|V_{i}-V_{j}\right|
$$

where, $\sigma_{i, j}$ is the gauge of capture, and moreover $\sigma_{i, j}$ is the symmetric positive function of the particles orders $[3,11]$.

We can find various models tasked to solve the problem of the aggregation matrix form [2, 4]. At the same time this problem has not been solved to this day. We think that the certain reasons can help to find corresponding model for elements of the aggregation matrix.

Intensive transition of components into the insoluble state is similar to the crystal origins arising in the supersaturated solutions. But in contrast to the real crystallization process in this case the particles of solid phase may be partially crystallized, i.e., the phase transition may be not completed $[2,5]$. However the active transfer to the insoluble phase proceeds at achievement of the critical value of concentration of the reagent which forms this insoluble phase.

The effect of increasing the effective gauge of capture for $i$-mers of low orders with growth of the characteristic particle radius plays the main role as well as decreasing the particles mobility with growth of their masses.

Thus we represented the model of aggregation matrix $\Phi_{i, j}$ in the form of certain even function of the special parameter 


$$
\lambda=\frac{i-j}{i+j}
$$

The numerical experiment has been carried out with the following matrix:

$$
\Phi_{i, j} \approx \frac{k}{(i+j)} \beta+a_{2}\left(\frac{i-j}{i+j}\right)^{2}
$$

Fig. 1 represents some results of this experiment. The presented model gave a correct qualitative description of the aggregation process, and the main conclusions confirmed the known data and results of asymptotic analysis $[2,12]$.

The intensive decrease of the monomers is observed at the initial stage of the process, and concentration of $i$-mers of highest orders increases at this stage. The concentration of orders closed to 2 passes through the maximum. Further we observe the stabilization of $i$-mers of certain orders. The specific order of $i$-mers corresponding to the maximum concentration depends on the parameters of the model (Eq. (10)).

It can be concluded that using the presented approach to describing the aggregation-fragmentation processes with model elements of aggregation matrix may be sufficiently relevant. Experimental data confirms the correctness of theoretical estimations. The presented model can be adapted to different physical-chemical systems and to different process regimes.

\section{Analysis of Results of Numerical Experiment}

Numerical experiment has been carried out with aggregation matrixes of a type (10) at various values of operating parametres for reactions of the first and second orders.

Some results of the spent experiment for reactions of the first order are shown in the Fig. 2.

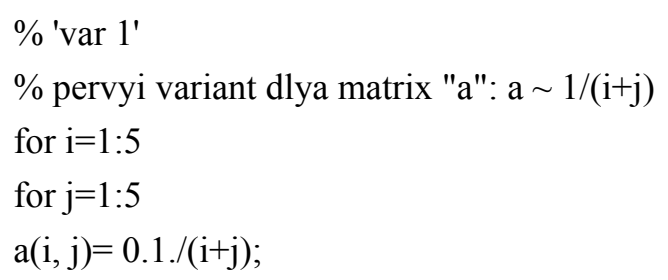

$\mathrm{I}=+\mathrm{k} 0 * \exp (-\mathrm{k} 1 * \mathrm{t})$

$\mathrm{k} 0=0.9$;

$\mathrm{k} 1=0.1$;

It is understood from Fig. 2, that process is characterized by fast growth of monomer concentration at the beginning of order $t \approx 2 \tau_{n}$, and then concentration of clusters of higher orders begin to prevail. Thus extreme values of concentrations of clusters of higher orders have clearly expressed time shifts.

On each set timing interval the certain stabilization of the average cluster order is observed. It follows, that for a reactor of periodic type for which the basic characteristic is reaction lead time, that means the time of initial reagents completion, it is necessary to know besides the general concentration of clusters of insoluble phase also the average meaning of clusters' order, because the average size of cluster determines

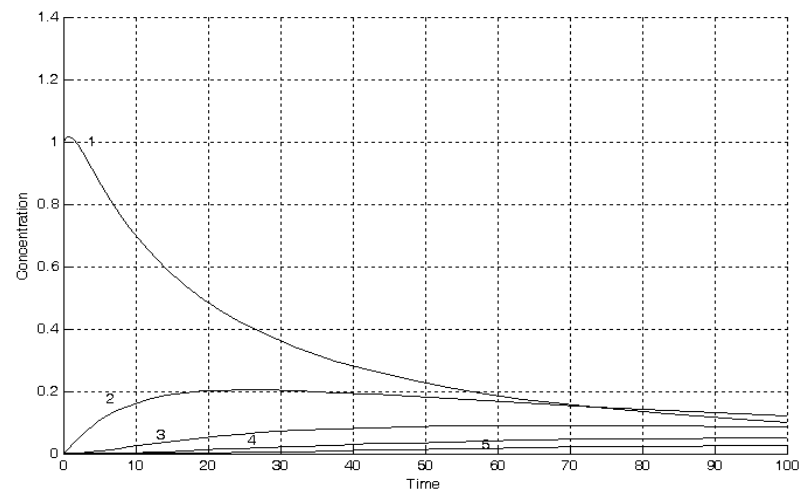

Fig. 1 Evolution of $i$-mers concentration according to the model (10) under $k=0,1 ; \beta=1 ; a_{2}=1$. Number of curve corresponds to the order $i$.

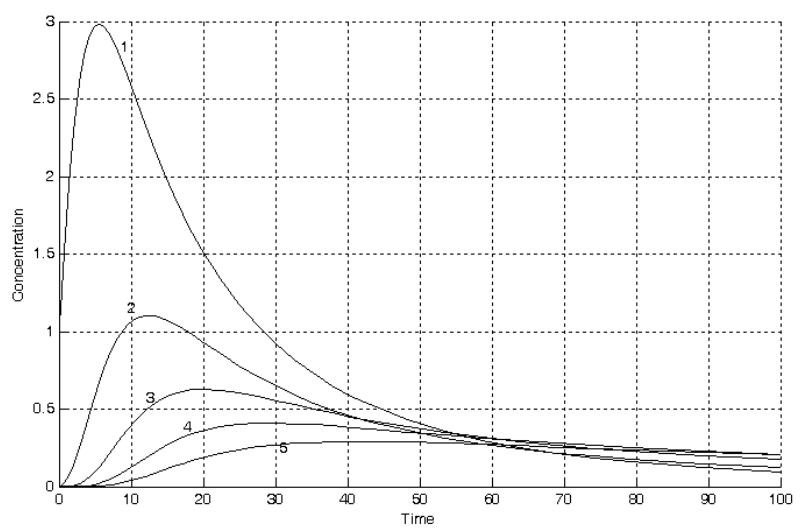

Fig. 2 Dependence of concentration of high order clusters from time. 
the deposition time and influences on work cycle of reactor.

As a result of numerical experiment data processing the following parity for calculation of average number of clusters is received:

$$
i_{c p}(t)=\frac{i_{\max }}{2}\left(1-\exp \left(-\frac{t}{\tau}\right)\right)
$$

The greatest interest, from the point of view of process kinetics in a reactor, represents the comparison of speed of change of the general number of clusters and clusters of the first order, formed as a result of primary nucleation of a reaction product.

In Fig. 3, some samples of the results of numerical experiments are shown. Researches were carried out for following values of operating parameters:

$\%$ 1-ya function: summa $=\mathrm{c}(\mathrm{j}, 1)+\mathrm{c}(\mathrm{j}, 2)+\mathrm{c}(\mathrm{j}, 3)+$ $\mathrm{c}(\mathrm{j}, 4)+\mathrm{c}(\mathrm{j}, 5)$

$\%$ 2-ya function: $\mathrm{y} 2=\mathrm{c}(\mathrm{j}, 1)$

On diagrams given below the red curves are constructed for function $(*)$, and all green curves are constructed for function $(* *)$.

For a monomer there is a source

$$
\mathrm{I}=+\mathrm{k} 0 * \exp (-\mathrm{k} 1 * \mathrm{t})
$$

From diagrams it is observed that the moment when speed of aggregation of monomers starts to prevail over speed of primary nucleation. It is a so-called threshold of weak coagulation $[6,9]$.

Another clearly visible important phenomenon is time shift of extrema of the curves describing monomers concentration and concentration of the general number of clusters of various orders. This shift causes the second stage of aggregation characterizing so-called strong aggregation $[7,8]$.

\section{Conclusions}

Modification of the Smoluhovsky equation for the description of aggregation of dispersions with a chemical source of monomers of insoluble phase is developed by taking into account the interaction of clusters of various orders. It is offered the generalized equation for the function, allowing, unlike the earlier

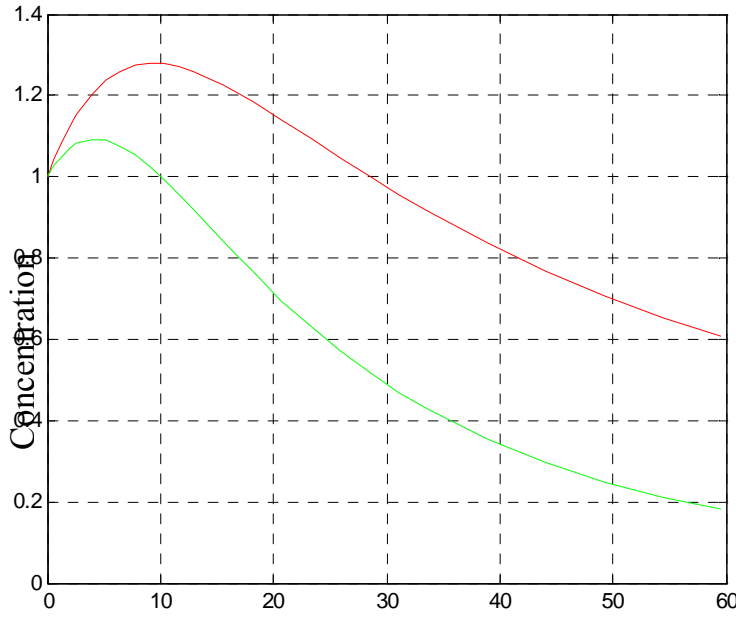

(a) $\mathrm{k} 0=0.1 ; \mathrm{k} 1=0.1$

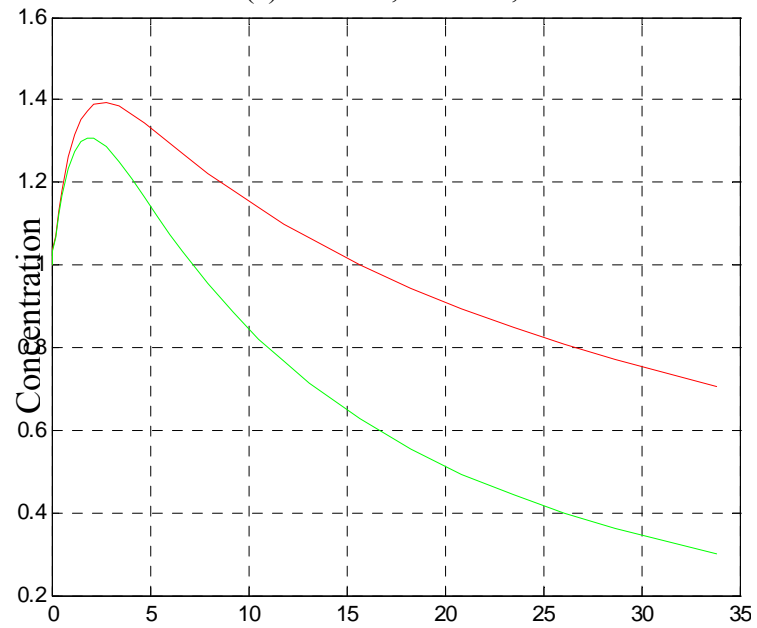

(b) $\mathrm{k} 0=0.5 ; \mathrm{k} 1=0.9$;

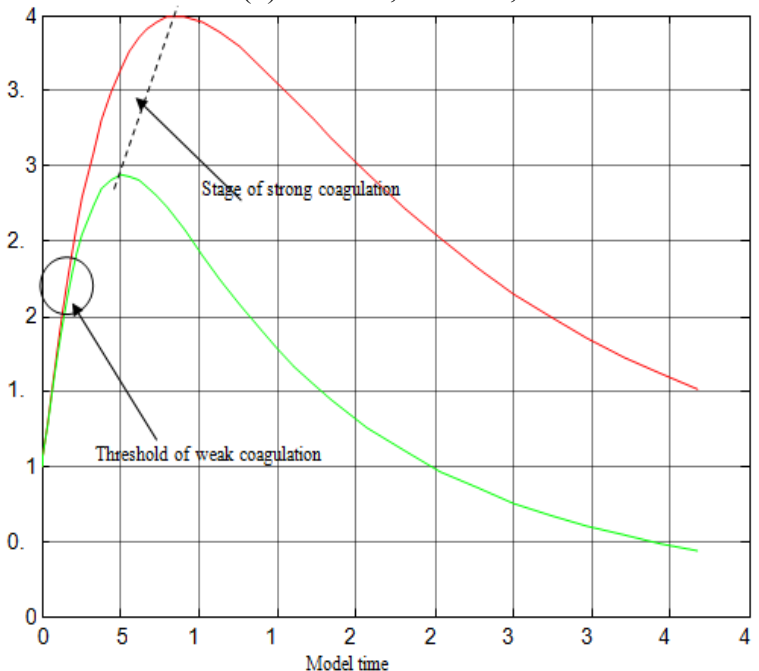

(c) $\mathrm{k} 0=0.9 ; \mathrm{k} 1=0.1 ; \mathrm{k} 0=0.9 ; \mathrm{k} 1=0.1$;

Fig. 3 (a) Dependence of concentration on modelling time at primary parametre $\mathrm{k0}=0.1 ; \mathrm{k1}=0.1$; (b) dependence of concentration on modelling time at primary parametre $\mathbf{k 0}=$ $0.5 ; \mathrm{k} 1=0.9$ and (c) analyses of results of numerical experiments for monomers. 
known method, to receive the analytical decision for calculation of total number of clusters in system with chemical sources of insoluble phase. Two critical values of concentration in the course of coagulation of insoluble phase in reacting systems are theoretically described and experimentally confirmed: the first crisis is caused by achievement of threshold of coagulation for the primary monomers, the second crisis is completion of active stage of chemical reaction in the periodic reactor and corresponding reduction of intensity of formation of monomers in system. The principle of determination of residence time in the periodic reactor with formation and sedimentation of insoluble phase in a working zone is formulated and experimentally confirmed.

\section{References}

[1] Sh.Z. Yeskendirov, Modeling and projecting of liquid-phase reactors of mixing, Shymkent: SKSU named after M. Auezov, 1995, p. 42.

[2] J.M. Ball, J. Carr, O. Penrose, The Becker-doring claster equations: basic properties and asymptotic behaviour of solutions, Commun. Math. Phys. 104 (1986) 657-692.

[3] D.B. Duncan, A.R. Soheili, Approximating the Becker-Dцring Cluster equations, Commun. Math. Phys. 119 (2000) 1-31.
[4] V.M. Voloschuk, Y.S. Sedunov, Processes of coagulation in disperse systems, Hydrometeoizdat., Leningrad, 1975, p. 320. (in Russian)

[5] J.A.D. Wattis, An introduction to mathematical models of coagulation-fragmentation processes: A discrete deterministic mean-field approach, Physica D: Nonlinear Phenomena 222 (1-2) (2006) 1-20.

[6] P. Jensen, A.L. Barabási, H. Larralde, S.. Havlin, H.E. Stanley, Model incorporating deposition, diffusion, and aggregation in submonolayer nanostructures, Phys. Rev. E. 50 (1994) (1) 618-621.

[7] J. Lee, S. Schwarzer, A. Coniglio, H.E. Stanley. Localization of growth sites in diffusion-limited-aggregation clusters: Multifractality and multiscaling, Phys. Rev 48 (2) (1993) 1305-1315.

[8] E. Sanz, C. Valeriani, D. Frenkel, M. Dijkstra, Evidence for out-of-equilibrium crystal nucleation in suspensions of oppositely charged colloids, [cond-mat.soft] 27 Jul 2007.

[9] P. Dominguez-Garcia, S. Melle, M.A. Rubioc, Morphology of anisotropic chains in a magnetorheological fluid during aggregation and disaggregation processes, Journal of Colloid and Interface Science 333 (2009) 221-229.

[10] Y. Farjoun, J. Neu, Exhaustion of nucleation in a closed system, Phys. Rev. E 78 (2008).

[11] B. Niethammer, A vanishing excess density limit of the Becker-Döring equations 159 (2004).

[12] I. Zaliapin, H. Wong, A. Gabrielov, Hierarchical aggregation in percolation model, Tectonophysics 413 (2006) 93-107. 